\title{
Solitary fibrous tumors of the spine: a pediatric case report with a comprehensive review of the literature
}

\author{
Gregory W. Albert, MD, ${ }^{1,2}$ and Murat Gokden, MD³ \\ 'Division of Neurosurgery, Arkansas Children's Hospital; and Departments of ${ }^{2}$ Neurosurgery and ${ }^{3}$ Pathology, University of \\ Arkansas for Medical Sciences, Little Rock, Arkansas \\ Solitary fibrous tumors of the spine are rare lesions. Their description in the literature is limited to case reports and small \\ case series. While generally benign lesions, they can recur and occasionally occur as malignancies. Here the authors \\ present the case of a 10-year-old boy, the youngest patient and first preadolescent reported thus far, with this condition. \\ In addition, they perform a comprehensive review of all previously published cases of spinal solitary fibrous tumors. \\ https://thejns.org/doi/abs/10.3171/2016.10.PEDS16279
}

KEY WORDS solitary fibrous tumor; spine; central nervous system; pediatric; oncology

$\mathrm{S}$ OLITARY fibrous tumors (SFTs) were initially described by Klemperer and Rabin in 1931. ${ }^{36}$ These lesions are most commonly found in the pleura and abdomen/pelvis. One large single-center review suggests a nearly even split between benign and malignant pathologies. ${ }^{19}$ A recent review of the Surveillance, Epidemiology, and End Results (SEER) database found 804 cases reported over nearly 40 years. Tumors in these patients were thoracic/abdominal/pelvic in $35.3 \%$, central nervous system (CNS) in $24.5 \%$, head/neck in $19.9 \%$, and extremity in $16.2 \%$. Most reported cases did not include pathological classification, and a plurality of patients (49.4\%) underwent surgery without adjuvant therapy. ${ }^{79}$

Carneiro and colleagues described the first 2 cases of spinal SFT, both of which were treated with surgery. ${ }^{12}$ Few comprehensive reviews of these lesions exist. Bisceglia et al. found 220 cases of CNS SFTs, 50 of which occurred in the spine. ${ }^{5}$ Fargen et al. reviewed 189 cases of CNS SFTs, including 46 spinal SFTs, and added 1 other spinal SFT case from their own experience. ${ }^{22}$

Spinal SFT in pediatric patients is quite rare. The youngest patient with spinal SFT reported thus far was a 17-year-old male, ${ }^{30}$ and there have been only 2 other previously reported cases of spinal SFT in patients younger than 21 years of age. ${ }^{11,71}$ Here we describe the case of an intradural extramedullary spinal SFT in a 10-year-old boy. In addition, we identified 81 unique patients with spinal SFT in the literature and comprehensively review these reports.

\section{Case Report}

History and Examination

A 10-year-old, previously healthy male was admitted to the neurology service for evaluation of right upper extremity weakness. On examination, he was unable to adduct his right shoulder and had Medical Research Council Grade 4/5 strength in the other muscle groups of his right upper extremity. Otherwise, his motor and sensory examinations were normal. He was hyperreflexic in the right upper and lower extremities with a positive Babinski sign on the right. Magnetic resonance imaging studies revealed an intradural extramedullary tumor on the right side at $\mathrm{C} 1-3$ that was hypointense on T1- and T2-weighted sequences with homogeneous enhancement (Fig. 1A-C). Preoperative diagnosis was meningioma or, less likely, nerve sheath tumor.

\section{Operation}

The patient was taken to surgery where a C1-3 lami- 

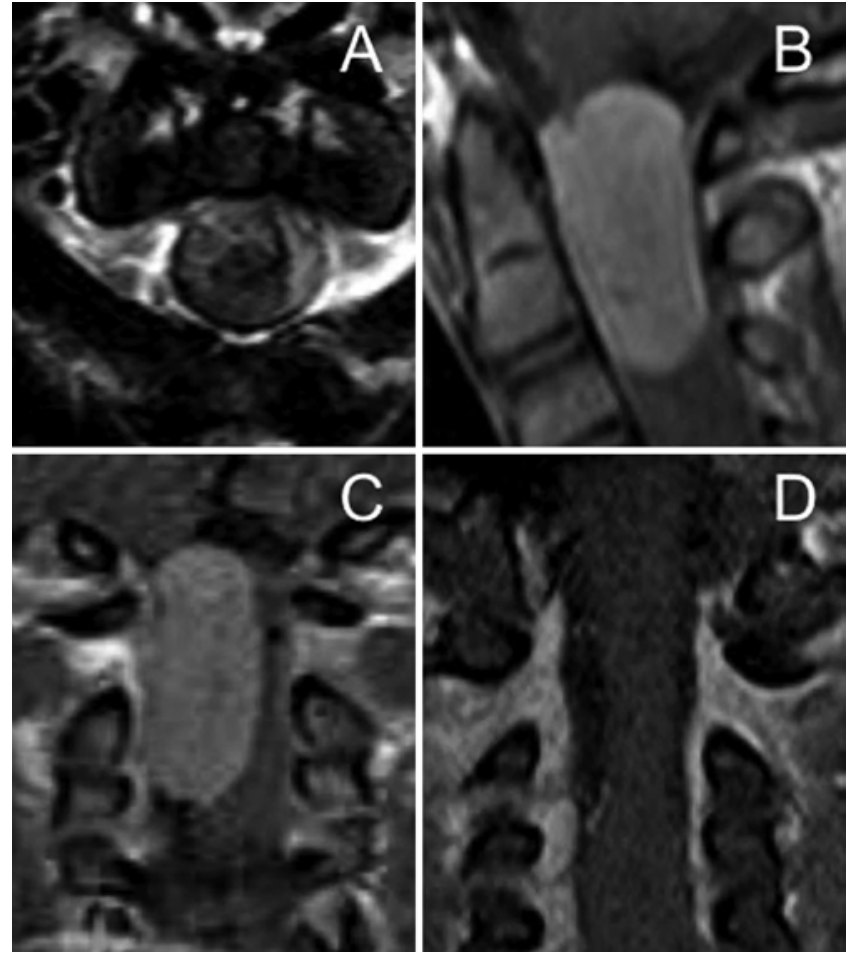

FIG. 1. Preoperative axial T2-weighted MR image (A) demonstrating hypointensity of the lesion and cord compression. Preoperative sagittal (B) and coronal (C) T1-weighted MR images with contrast demonstrating homogeneous enhancement of the tumor. Postoperative coronal T1weighted MR image (D) with contrast demonstrating GTR and reexpansion of the spinal cord.

nectomy was performed for excision of the tumor. After performing two-thirds laminectomies at each level, a paramedian durotomy was made. The firm, rubbery pale tumor was intradural extramedullary without dural attachment. It was located between the dorsal and ventral nerve rootlets. It was adherent to the lateral aspect of the spinal cord but could be dissected free of pia mater without injury to the cord and did not have an intramedullary component. The tumor had a pial attachment at its caudal end. The tumor also appeared to be attached to the right dentate ligament at C-1. Gross-total resection (GTR) was achieved in a piecemeal fashion (Fig. 2). There were no intraoperative complications.

\section{Postoperative Course}

Postoperative MRI confirmed GTR (Fig. 1D). The patient was discharged home on postoperative Day 4 with outpatient physical therapy. At the time of discharge, he had trace residual weakness in his right hand and otherwise had full strength with no new neurological deficits. At 4.5 months after surgery, he had regained full strength, MRI showed no evidence of recurrent disease, and dynamic cervical spine radiographs showed no evidence of instability (Fig. 3). One year after surgery, the patient had a normal neurological examination, no evidence of recurrence on MRI, and maintained stability on flexion and extension radiographs of the cervical spine.

Light microscopy examination of the tissue showed a
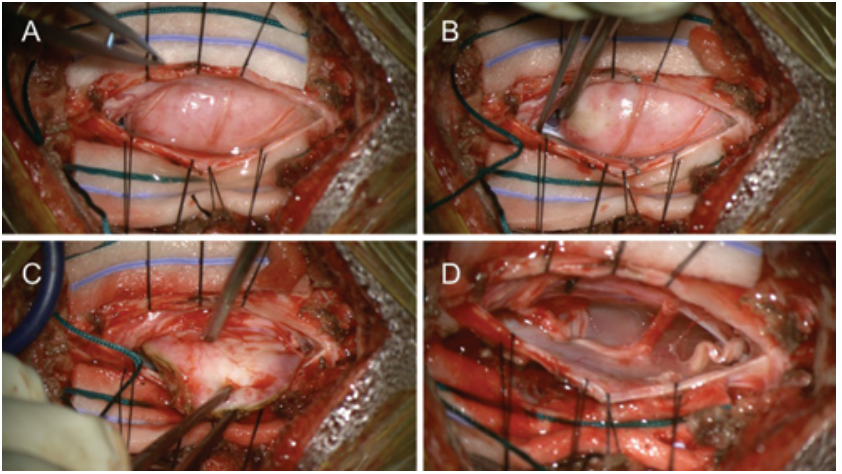

FIG. 2. Intraoperative photographs of tumor resection. A: Initial view of tumor with dorsal nerve roots overlying the lesion. B: Pial attachment at caudal end of tumor. C: Attachment of tumor to pia of spinal cord. D: Tumor has been completely resected. Figure is available in color online only.

spindle cell neoplasm with alternating cellular and collagenous areas. A few blood vessels were well demarcated with thin walls and showed branching, that is, staghorn vessels (Fig. 4A-C). A few nuclei had nucleoli, but the cell population was overall monomorphous without significant cytological atypia. Mitotic count was variable between 1 and 5/10 hpf on multiple counts in different areas. No necrosis, hemorrhage, or hypercellularity was seen. The neoplasm was diffusely and strongly positive for Bcl-2 and CD34 (Fig. 4D) with a diffuse nuclear staining for STAT6 (Fig. 4E). The Ki-67 proliferation index was variable between $10 \%$ and $15 \%$ in various areas (Fig. 4F). Epithelial membrane antigen and S100 protein were negative. This appearance was consistent with SFT with atypical features.

\section{Review of the Literature}

A PubMed search was performed for SFTs of the spine. In addition, the reference section of each paper was reviewed, allowing for the identification of additional cases. We identified 65 previously published papers with 83 cases. ${ }^{1-4,7-14,16,17,20-22,24-35,37-40,42,43,46-59,62,64-74,76,78,80,81}$ Two of these papers clearly duplicated patients (4 reports of 2 unique patients). ${ }^{14,76}$ A third reported patient was a suspected duplication. ${ }^{12,57}$ Eliminating these duplications and adding our own report yielded 82 reported cases of spinal SFT (Table 1).

Seventy-eight cases reported age at presentation. The majority of these cases occurred in adults $(94.8 \%$ in patients over the age of 21 years). Patients ranged in age from 10 years (our patient) to 83 years (median 51.5 years). Seventy-eight cases contained information on patient sex. There is a slight male predominance in the reported cases of spinal SFT (56.4\% male vs $43.6 \%$ female; Table 2$)$.

Seventy-six reported cases indicated spinal level. The plurality of lesions occurred in the thoracic region (42.1\%), followed by the cervical region $(27.6 \%)$ and lumbar region $(13.2 \%)$. The remaining tumors occurred in the sacrum, ${ }^{50}$ across junctional levels, ${ }^{1,11,13,22,28,46}$ or at multiple levels. ${ }^{35,47,67,68}$ Sixty-eight reported cases contained information on the spinal compartment involved. The plurality 

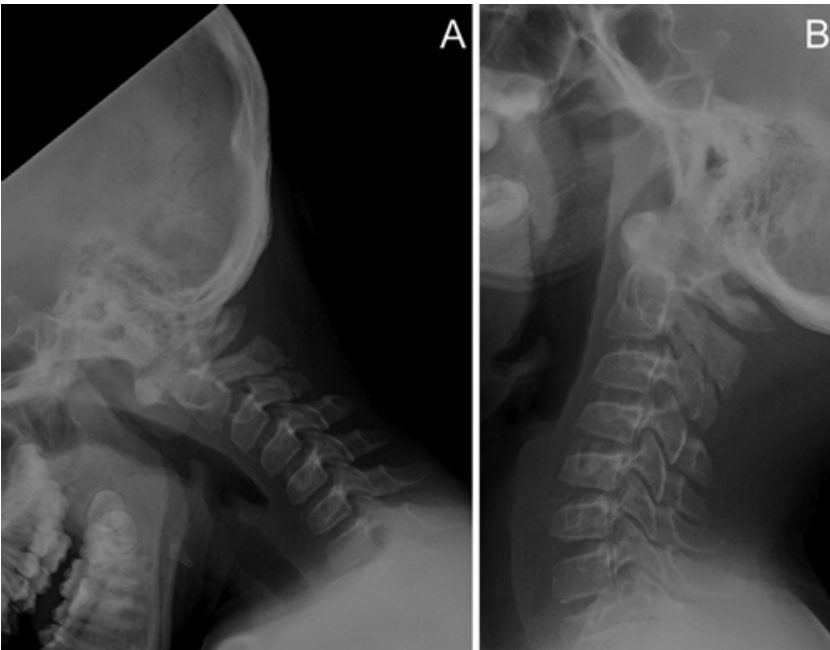

FIG. 3. Postoperative flexion (A) and extension (B) cervical spine radiographs demonstrating no evidence of dynamic instability.

(35.3\%) was intradural extramedullary, followed by extradural $(26.5 \%)$. Of these extradural tumors, 6 were primarily extraspinal or bone as opposed to primarily intraconal (Table 2). ${ }^{27,31,40,51-53}$

Sixty-one reported cases contained information on MRI appearance. Tumors in all of these cases exhibited some degree of enhancement with intravenous contrast, most of them homogeneously enhancing. Most of the lesions were either hypointense or isointense on T1- and T2-weighted imaging, although rare tumors were hyperintense on one or the other of these sequences. A few reports indicated preoperative diagnoses, most often meningioma or nerve sheath tumor., $2,10,43,64,65,72,73$ One hemorrhagic lesion in the conus was thought to be either a myxopapillary ependymoma or cavernous malformation..$^{74}$

Seventy-five of the reported cases contained information regarding treatment. Patients in all of these cases underwent surgery. Gross-total resection was achieved in $82.7 \%$ of patients, and subtotal resection (STR) was performed in $17.3 \%$. Four patients (3 GTR and 1 STR) underwent preoperative embolization of the tumor.,90,53,67 One GTR patient also underwent intraoperative embolization. ${ }^{71}$ Two of the GTR outcomes were achieved in 2 stages of surgery. ${ }^{10,51}$ Four patients (3 GTR and 1 STR) required spinal fusion at the initial presentation. ${ }^{26,31,51,71} \mathrm{~A}$ single patient with a malignant SFT was treated with chemotherapy and radiation after STR. ${ }^{67}$ Otherwise, chemotherapy and radiation were reserved for recurrences and/or metastases. $9,26,46,50,51$

Forty-seven patients had reports of intraoperative assessments of tumor origin. In most cases, the origin was either the spinal cord or the dura mater, with fewer reports of tumors arising from nerve roots. Four patients, including our own, had attachment to the pia mater. ${ }^{3,9,29}$ Four patients had tumors arising from bone or periosteum, $26,52,67,71$ 2 patients had tumors involving the arachnoid, ${ }^{10,22} 1$ patient's tumor seemed to arise from the venous plexus. ${ }^{27}$

Seventy-eight cases indicated tumor histopathology. Sixty-nine patients (92\%) had SFT without mention of malignant or other unusual features on pathology. Five

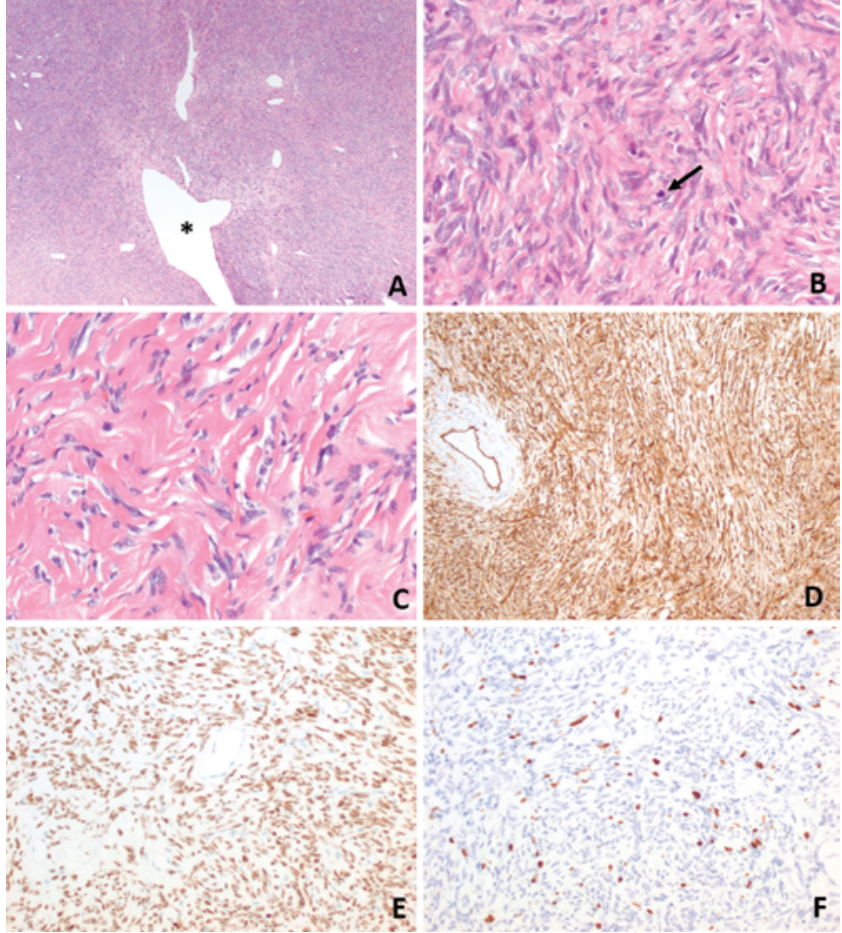

FIG. 4. Microscopic findings. A: Low-power view with peculiar, welldefined, branching blood vessels (asterisk) and alternating dark and light areas reflecting variable cellularity and collagen. B: Spindle cell morphology of the neoplastic cells and mitotic activity (arrow). C: Thick, keloid-like collagen bundles and decreased cellularity in some areas. D: Diffuse and strong CD34 positivity. E: Nuclear STAT6 expression. F: Higher-than-expected Ki-67 proliferation index. $\mathrm{H}$ \& $\mathrm{E}$ $(A-C)$, original magnifications $\times 40(A), \times 200(B-C), \times 100(D-F)$. Figure is available in color online only.

patients (6.7\%) had malignant SFT. ${ }^{924,35,51,67}$ Three of the previously reported cases included unusual pathological findings such as retiform or papillary features ${ }^{70}$ focal pleomorphism, ${ }^{7}$ and lipomatous features. ${ }^{1}$ Our patient's tumor demonstrated atypical features without reaching the criteria for malignant SFT.

Outcome depended largely on histology and extent of resection. Four of the patients with malignant SFT had outcome data reported. All 4 died of their disease, one at 2 months, ${ }^{24}$ one at 6 months, ${ }^{67}$ one at 9 months,,${ }^{51}$ and one at 4 years. ${ }^{9}$ Fifty-six patients with SFT had outcome data reported. Follow-up times ranged from 2 months to 21 years. Twenty-two patients had follow-up of 1 year or less, and 45 patients had follow-up of 5 years or less. Outcome of SFT without malignant features seemed to depend largely on extent of resection. Nine SFT patients with STR had information on outcome. Three of these patients had only 6 months of follow-up and no recurrence or progression..$^{28,34,62}$ Of the remaining 6 patients, 5 exhibited local disease progression between 6 months and 21 years after the first presentation. $8,12,26,37,46$ The final patient developed a new spinal lesion 14 years after the original surgery. ${ }^{56}$ Forty-six patients treated with GTR had data on outcome and recurrence. Of these patients, $41(89.1 \%)$ had no recurrence at a median of 1.2 years of follow-up (range 2 months -7 years). One of these patients had residual dis- 
TABLE 1. Literature summary of spinal SFT cases

\begin{tabular}{|c|c|c|c|c|c|c|c|c|}
\hline Authors \& Year & $\begin{array}{l}\text { Age } \\
\text { (yrs)/ } \\
\text { Sex }\end{array}$ & Level & Compartment & Treatment & Histology & $\begin{array}{l}\text { Last } \\
\text { FU } \\
\text { (yrs) }\end{array}$ & Outcome & Notes \\
\hline \multirow[t]{2}{*}{ Carneiro et al., 1996} & $50 / F$ & NR & IDEM \& IDIM & STR & SFT & 6.0 & Recurrence & $\begin{array}{l}\text { Died } 1 \text { wk after sur- } \\
\text { gery for recurrence }\end{array}$ \\
\hline & $54 / F$ & Lumbar & NR & GTR & SFT & 7.0 & Disease free & $\begin{array}{l}\text { Died of unrelated } \\
\text { causes }\end{array}$ \\
\hline Alston et al., 1997 & $47 / \mathrm{M}$ & $\mathrm{T} 4-5$ & IDIM & GTR & SFT & 0.2 & Disease free & \\
\hline Kuchelmeister et al., 1997 & $64 / \mathrm{M}$ & C-5 & IDEM \& IDIM & GTR & SFT & NR & NR & \\
\hline Malek et al., 1997 & $33 / \mathrm{M}$ & T7-8 & IDEM & GTR & SFT & NR & NR & \\
\hline \multirow[t]{2}{*}{ Brunori et al., 1999} & $18 / \mathrm{M}$ & $\mathrm{O}-\mathrm{C} 3$ & ED \& IDEM & GTR & SFT & 1.0 & Disease free & \\
\hline & $46 / F$ & T12-L1 & IDEM & GTR & SFT & 0.3 & Disease free & \\
\hline Hasegawa et al., 1999 & $39 / \mathrm{M}$ & NR & NR & GTR & SFT & 9.0 & $\begin{array}{l}\text { Local recurrence at } \\
5 \text { yrs \& } 9 \text { yrs }\end{array}$ & \\
\hline \multirow[t]{2}{*}{ Kanahara et al., 1999} & $62 / M$ & C6-7 & IDEM \& IDIM & Surgery & SFT & NR & NR & \\
\hline & $46 / F$ & $\mathrm{C} 4-5$ & ED \& IDEM & GTR & SFT & NR & NR & \\
\hline Donnellan et al., 2000 & $39 / \mathrm{M}$ & $\mathrm{L}-1$ & ED & GTR & SFT & NR & NR & \\
\hline Mordani et al., 2000 & $33 / \mathrm{M}$ & C-5 & IDIM & GTR & SFT & 1.5 & Disease free & \\
\hline Vorster et al., 2000 & $51 / \mathrm{M}$ & T2-3 & IDEM & GTR & SFT & 0.6 & Disease free & \\
\hline Kurtkaya et al., 2001 & $70 / F$ & T-3 & IDEM & GTR & SFT & 1.0 & Disease free & \\
\hline Endo et al., 2003 & $63 / F$ & C2-4 & ED & GTR & SFT & NR & NR & Dumbbell tumor \\
\hline Obara et al., 2003 & $49 / F$ & C2-4 & ED & $\begin{array}{l}\text { GTR + preop } \\
\text { embo }\end{array}$ & SFT & 1.0 & Disease free & Primarily extraspinal \\
\hline \multirow[t]{4}{*}{ Tihan et al., 2003} & NR & NR & IDIM & NR & NR & NR & NR & \\
\hline & NR & NR & IDIM & NR & NR & NR & NR & \\
\hline & NR & NR & IDEM & NR & NR & NR & NR & \\
\hline & NR & NR & IDEM & NR & NR & NR & NR & \\
\hline Bohinski et al., 2004 & $49 / F$ & C-4 & IDEM \& IDIM & GTR & $\begin{array}{l}\text { SFT w/ focal } \\
\text { pleomorphism }\end{array}$ & 0.8 & Disease free & \\
\hline Caroli et al., 2004 & $54 / \mathrm{M}$ & $\mathrm{C} 7-\mathrm{T} 1$ & IDEM & GTR & SFT & 1.3 & Disease free & \\
\hline Hirakawa et al., 2004 & $52 / \mathrm{M}$ & C1-2 & ED & GTR & SFT & 2.5 & Disease free & Primarily extraspinal \\
\hline Kawamura et al., 2004 & $64 / M$ & T2-3 & IDEM \& IDIM & STR & SFT & 0.5 & No progression & \\
\hline Kim et al., 2004 & $55 / \mathrm{M}$ & $\begin{array}{l}\text { C-6, T-3, } \\
\text { T4-5, } \\
\text { L-5, S-2 }\end{array}$ & ED & STR & Malignant SFT & NR & NR & $\begin{array}{l}\text { Prior posterior fossa } \\
\quad \text { tumor }\end{array}$ \\
\hline Piana et al., 2004 & $67 / \mathrm{M}$ & L1-2 & ED & GTR & SFT & NR & NR & \\
\hline \multirow[t]{2}{*}{ Pizzolitto et al., 2004} & $36 / \mathrm{M}$ & T7-8 & IDEM & GTR & SFT & 1.5 & Disease free & \\
\hline & $47 / \mathrm{M}$ & C-4 & IDIM & GTR & SFT & 1.0 & Disease free & \\
\hline Miyashita et al., 2004 & $63 / F$ & C-7, T3-5 & IDEM & GTR (1 lesion) & SFT & NR & Stable residue & $\begin{array}{l}\text { T-5 lesion resected, } \\
\text { had tentorial lesion } \\
15 \text { yrs prior }\end{array}$ \\
\hline \multirow[t]{4}{*}{ Jallo et al., 2005} & $41 / \mathrm{M}$ & C6-7 & IDIM & GTR & SFT & 3.5 & Disease free & \\
\hline & $17 / \mathrm{M}$ & T-5, T-6 & IDIM & GTR & SFT & 1.6 & Disease free & 2 separate lesions \\
\hline & $59 / \mathrm{M}$ & T-5 & NR & GTR & SFT & 4.8 & Disease free & \\
\hline & $37 / F$ & T2-3 & NR & GTR & SFT & 5.0 & Disease free & \\
\hline Ogawa et al., 2005 & $63 / F$ & $\mathrm{~T}-11$ & IDEM & GTR & SFT & NR & NR & \\
\hline Ogungbo et al., 2005 & $53 / \mathrm{M}$ & C3-4 & IDEM \& IDIM & GTR & SFT & NR & NR & \\
\hline Pakasa et al., 2005 & $27 / \mathrm{M}$ & T5-7 & IDEM \& IDIM & STR & SFT & 14.0 & $\begin{array}{l}\text { New sacral lesion } \\
\text { at } 14 \text { yrs treated } \\
\text { w/ GTR }\end{array}$ & \\
\hline
\end{tabular}


» CONTINUED FROM PAGE 342

TABLE 1. Literature summary of spinal SFT cases

\begin{tabular}{|c|c|c|c|c|c|c|c|c|}
\hline Authors \& Year & $\begin{array}{l}\text { Age } \\
\text { (yrs)/ } \\
\text { Sex }\end{array}$ & Level & Compartment & Treatment & Histology & $\begin{array}{l}\text { Last } \\
\text { FU } \\
\text { (yrs) }\end{array}$ & Outcome & Notes \\
\hline \multirow[t]{6}{*}{ Metellus et al., 2007} & $56 / M$ & $\mathrm{C} 7-\mathrm{T} 1$ & NR & STR & SFT & 3.5 & $\begin{array}{l}\text { Local progression } \\
\text { at } 2 \text { yrs treated } \\
\text { w/ GTR }\end{array}$ & \\
\hline & $57 / M$ & L-2 & NR & GTR & SFT & 8.5 & $\begin{array}{l}\text { Recurred at } 8.5 \text { yrs, } \\
\text { treated w/ GTR \& } \\
\text { chemo }\end{array}$ & \\
\hline & $57 / M$ & T-9 & NR & GTR & SFT & 1.8 & Disease free & \\
\hline & $44 / F$ & $\mathrm{~T}-12$ & NR & GTR & SFT & 4.2 & Disease free & \\
\hline & $72 / F$ & $\mathrm{~T}-12$ & NR & GTR & SFT & 0.8 & Disease free & \\
\hline & $44 / F$ & T-7 & NR & GTR & SFT & 1.7 & Disease free & \\
\hline Nakamura et al., 2007 & $28 / F$ & Cervical & ED & GTR & SFT & 5.0 & Disease free & $\begin{array}{l}\text { Arising dorsally from } \\
\text { lamina \& facets }\end{array}$ \\
\hline Hashimoto et al., 2008 & $71 / F$ & C4-5 & ED & $\begin{array}{l}\text { STR + anterior } \\
\quad \text { fusion }\end{array}$ & SFT & 4.0 & $\begin{array}{l}\text { Progressed at } 6 \\
\text { mos \& } 2.5 \text { yrs }\end{array}$ & $\begin{array}{l}\text { Required additional } \\
\text { resection \& fusion } \\
\text { surgeries }\end{array}$ \\
\hline Muñoz et al., 2008 & $35 / \mathrm{M}$ & Sacral & NR & GTR & SFT & 8.0 & $\begin{array}{l}\text { Local recurrence w/ } \\
\text { pulmonary mets } \\
\text { at } 5.5 \text { yrs }\end{array}$ & $\begin{array}{l}\text { Malignant pulmonary } \\
\text { mets at } 3.5 \mathrm{yrs} \text {; } \\
\text { pulmonary/hepatic } \\
\text { mets at } 6.5 \mathrm{yrs}\end{array}$ \\
\hline Shin et al., 2008 & $40 / F$ & C6-7 & ED \& IDEM & GTR & SFT & 0.7 & Disease free & Dumbbell tumor \\
\hline Arantes et al., 2009 & $22 / \mathrm{M}$ & $\mathrm{T} 1-2$ & IDEM & GTR & SFT & 1.5 & Disease free & \\
\hline Ciappetta et al., 2010 & $75 / F$ & T6-7 & IDEM \& IDIM & GTR & SFT & 2.0 & Disease free & \\
\hline Ishii et al., 2009 & $63 / F$ & C-5 & IDIM & GTR & SFT & 1.2 & Disease free & \\
\hline Kakimaru et al., 2009 & $75 / F$ & T8-10 & ED & $\begin{array}{l}\text { GTR + dorsal } \\
\text { fusion }\end{array}$ & SFT & 3.0 & Disease free & $\begin{array}{l}\text { Dumbbell tumor w/ } \\
\text { large extraspinal } \\
\text { component }\end{array}$ \\
\hline Aftab et al., 2010 & $38 / \mathrm{M}$ & L4-S2 & ED & STR & $\begin{array}{l}\text { SFT w/ lipoma- } \\
\text { tous features }\end{array}$ & 0.4 & Stable residue & \\
\hline Cincu et al., 2010 & $58 / \mathrm{M}$ & T3-5 & IDEM & GTR & SFT & NR & NR & \\
\hline Vassal et al., 2011 & $52 / F$ & T8-9 & ED \& IDEM & GTR & SFT & 5.2 & Disease free & \\
\hline \multirow[t]{2}{*}{ Fargen et al., 2011} & $62 / \mathrm{M}$ & $\mathrm{O}-\mathrm{C} 2$ & IDEM & GTR & SFT & 0.5 & Disease free & \\
\hline & $28 / F$ & C2-3 & IDEM \& IDIM & GTR & SFT & 2.0 & $\begin{array}{l}\text { Small radiographic } \\
\text { recurrence }\end{array}$ & Lost to FU \\
\hline Guo et al., 2012 & $60 / M$ & Thoracic & Intracanal & Surgery & Malignant SFT & 0.2 & $\begin{array}{l}\text { Deceased from } \\
\text { metastatic } \\
\text { disease }\end{array}$ & $\begin{array}{l}\text { Metastatic from renal } \\
\text { primary }\end{array}$ \\
\hline Takenouchi et al., 2011 & $45 / F$ & $\begin{array}{c}\text { C-3, C7- } \\
\text { T1 }\end{array}$ & IDEM & GTR (1 lesion) & SFT & 0.4 & Stable residue & $\begin{array}{l}\text { Prior posterior fossa } \\
\text { tumor }\end{array}$ \\
\hline Bouyer et al., 2012 & $56 / F$ & T10-11 & ED & STR & SFT & 5.0 & $\begin{array}{l}\text { Progressed at } 6 \\
\text { mos, recurred at } \\
4 \text { yrs }\end{array}$ & \\
\hline \multirow[t]{2}{*}{ Mariniello et al., 2012} & $67 / M$ & C4-7 & IDEM & GTR & SFT & 1.0 & Disease free & \\
\hline & $75 / F$ & T6-7 & IDEM & GTR & SFT & 1.0 & Disease free & \\
\hline Wu et al., 2012 & $50 / F$ & $L-2$ & IDEM & GTR & SFT & NR & NR & $\begin{array}{l}\text { Arising from filum } \\
\text { terminale }\end{array}$ \\
\hline \multirow[t]{2}{*}{ Chen et al., 2012} & $43 / \mathrm{M}$ & T11-12 & NR & GTR & SFT & 14.7 & Recurred at $14.7 \mathrm{yrs}$ & \\
\hline & $63 / \mathrm{M}$ & L4-S2 & NR & GTR & SFT & 2.8 & Disease free & \\
\hline Brigui et al., 2013 & $56 / \mathrm{M}$ & T6-7 & IDEM & GTR & SFT & 2.4 & Disease free & \\
\hline
\end{tabular}


» CONTINUED FROM PAGE 343

TABLE 1. Literature summary of spinal SFT cases

\begin{tabular}{|c|c|c|c|c|c|c|c|c|}
\hline Authors \& Year & $\begin{array}{l}\text { Age } \\
\text { (yrs)/ } \\
\text { Sex }\end{array}$ & Level & Compartment & Treatment & Histology & $\begin{array}{l}\text { Last } \\
\text { FU } \\
\text { (yrs) }\end{array}$ & Outcome & Notes \\
\hline Brigui et al., 2013 & $69 / \mathrm{M}$ & T7-8 & ED & $\begin{array}{l}\text { GTR + preop } \\
\text { embo }\end{array}$ & Malignant SFT & 4.0 & $\begin{array}{l}\text { Deceased from } \\
\text { metastatic } \\
\text { disease }\end{array}$ & $\begin{array}{l}\text { Primary pulmonary } \\
\text { lesions; developed } \\
\text { kidney/extremity/ } \\
\text { visceral mets }\end{array}$ \\
\hline Montano et al., 2013 & $56 / F$ & L1-2 & IDEM & GTR & SFT & 1.0 & Disease free & \\
\hline Son et al., 2013 & $54 / F$ & T-8, S1-2 & ED & $\begin{array}{l}\text { STR + preop } \\
\text { embo + } \\
\text { postop radia- } \\
\text { tion/chemo }\end{array}$ & Malignant SFT & 0.5 & $\begin{array}{l}\text { Deceased from } \\
\text { metastatic } \\
\text { disease }\end{array}$ & $\begin{array}{l}\text { Presented w/ multifo- } \\
\text { cal disease (skull, } \\
\text { spine, pelvis) }\end{array}$ \\
\hline Tomek et al., 2013 & $66 / \mathrm{M}$ & $\mathrm{T} 11-12$ & ED & STR & $\begin{array}{l}\text { SFT w/ retiform } \\
\text { \& papillary } \\
\text { features }\end{array}$ & 4.1 & $\begin{array}{l}\text { Progressed at } 18 \\
\text { mos }\end{array}$ & \\
\hline Hwang et al., 2014 & $48 / \mathrm{M}$ & $\mathrm{C} 7-\mathrm{T} 1$ & IDEM & STR & SFT & 0.5 & No progression & \\
\hline Kobayashi et al., 2014 & $40 / \mathrm{M}$ & $C 4-5$ & IDEM \& IDIM & STR & SFT & 21.0 & $\begin{array}{l}\text { Progression at } 21 \\
\text { yrs }\end{array}$ & \\
\hline Nagano et al., 2014 & $57 / F$ & L4-5 & ED & $\begin{array}{l}\text { GTR + fusion (2 } \\
\text { stages) }\end{array}$ & Malignant SFT & 0.8 & $\begin{array}{l}\text { Deceased from } \\
\text { metastatic } \\
\text { disease }\end{array}$ & $\begin{array}{l}\text { Local \& metastatic } \\
\text { recurrence at } 3 \\
\text { mos }\end{array}$ \\
\hline Robert et al., 2014 & $49 / F$ & T9-10 & IDEM \& IDIM & STR & SFT & 0.5 & No progression & \\
\hline Sebaaly et al., 2014 & $32 / F$ & L3-4 & IDEM & GTR & SFT & 1.3 & Disease free & \\
\hline Tsutsumi et al., 2014 & 19/M & $C-1$ & ED & $\begin{array}{l}\text { GTR + intraop } \\
\text { embo }+ \\
\text { fusion }\end{array}$ & SFT & 1.0 & Disease free & $\begin{array}{l}\text { Primarily bone, had } \\
\text { parapharyngeal } \\
\text { lesion } 10 \text { yrs prior }\end{array}$ \\
\hline Yuan et al., 2014 & $48 / \mathrm{M}$ & T-9 & ED \& IDEM & GTR & SFT & NR & NR & Dumbbell tumor \\
\hline Basaran et al., 2015 & $67 / M$ & L-3 & IDEM & GTR & SFT & 1.0 & Disease free & \\
\hline Bruder et al., 2015 & $83 / F$ & T8-9 & IDIM & GTR (2 stages) & SFT & 0.7 & Disease free & \\
\hline Sade et al., 2015 & $43 / \mathrm{M}$ & Thoracic & IDEM & Surgery & SFT & NR & NR & \\
\hline Walker et al., 2015 & $47 / F$ & $L-1$ & IDIM & $\begin{array}{l}\text { GTR + preop } \\
\text { embo }\end{array}$ & SFT & 0.5 & Disease free & $\begin{array}{l}\text { Hemorrhagic lesion in } \\
\text { conus medullaris }\end{array}$ \\
\hline Lavrador et al., 2015 & $23 / F$ & $\mathrm{~T} 11-12$ & ED & $\begin{array}{l}\text { GTR + preop } \\
\text { embo }\end{array}$ & SFT & 0.5 & Disease free & $\begin{array}{l}\text { Primarily extraspinal, } \\
\text { dumbbell }\end{array}$ \\
\hline $\begin{array}{l}\text { Zaldivar-Jolissaint et al., } \\
\quad 2016\end{array}$ & $33 / \mathrm{M}$ & C1-4 & ED & GTR & SFT & 7.0 & Disease free & \\
\hline Present case & 10/M & C1-3 & IDEM & GTR & $\begin{array}{l}\text { SFT w/ atypical } \\
\text { features }\end{array}$ & 1.0 & Disease free & \\
\hline
\end{tabular}

Chemo = chemotherapy; ED = extradural; embo = embolization; FU = follow-up; IDEM = intradural extramedullary; IDIM = intradural intramedullary; mets = metastasis; $\mathrm{NR}=$ not reported; $\mathrm{O}=$ occiput.

ease elsewhere but no recurrence of the resected lesion. ${ }^{68}$ Three patients had single local recurrences at 2 years, ${ }^{22} 8.5$ years, ${ }^{46}$ and 14.5 years. ${ }^{14}$ Another patient developed local recurrences twice, once at 5 years and again at 9 years. ${ }^{25}$ The final patient developed malignant transformation of his tumor with pulmonary metastases at 3.5 years and local spinal recurrence and recurrent pulmonary and hepatic metastases at 5.5 years. ${ }^{50}$

\section{Discussion}

Solitary fibrous tumors are lesions of mesenchymal origin that differentiate primarily along fibroblastic lines. Characteristically, they demonstrate spindle cells in a col- lagen background. They are now thought to exist along a spectrum with hemangiopericytomas, representing a more cellular and vascular subtype of the same tumor category. The majority of SFTs exhibit benign histology and behavior; however, malignancy does occur and is evidenced by a high mitotic count, increased cellularity, hemorrhage and/ or necrosis, pleomorphic nuclei, and foci of dedifferentiation. $^{18}$

A borderline increase in the mitotic rate and a high proliferation index indicate atypical features of the SFT in our patient. Malignancy in SFT is not well described, and the criteria are mainly developed for the other end of the spectrum, that is, hemangiopericytoma. ${ }^{23,44}$ Nonethe- 
less, a large series on soft tissue and pleural SFTs showed that patients 55 years of age or older with tumors $15 \mathrm{~cm}$ or larger and with a mitotic count of 4 or more per $10 \mathrm{hpf}$ had a high risk of metastasis and death, ${ }^{18}$ while a proliferation index of $10 \%$ or more was found to be associated with malignancy in another study. ${ }^{6}$ The importance of the proliferation index has been emphasized in predicting prognosis in a series of CNS SFTs,${ }^{14}$ as well as in a meta-analysis of CNS SFTs. ${ }^{5}$ Authors of the latter study concluded that a proliferation index of $5 \%$ or more may indicate a higher likelihood of recurrence. ${ }^{5}$

Solitary fibrous tumor was first described as a tumor arising from the pleura in the thoracic cavity. In this location, the majority of these tumors are benign. However, $10 \%-20 \%$ will exhibit locally aggressive behavior or malignancy. Surgery is the preferred method of treatment and carries with it a 5-year survival of close to $100 \%$. Adjuvant therapy is reserved for metastatic or recurrent disease. Chemotherapy and radiation are only moderately effective. In the thorax, most tumors that recur do so within 2 years, and imaging is recommended every 6 months for 2 years followed by annual imaging. Malignant SFT carries a poor prognosis, with $63 \%$ of tumors recurring despite GTR and most patients dying within 2 years of diagnosis. ${ }^{15}$

A recent single-center review of 82 patients with SFT demonstrated a slight female predominance (57\%). ${ }^{19}$ Among this study cohort there was a nearly even split between benign and malignant histologies. However, patients were identified through a search of a sarcoma database at a tertiary care cancer center that likely skewed the series toward a malignant profile. The median survival of patents with benign tumors was 15.5 years with a 5-year survival of just over $75 \%$. The median survival of patients with malignant tumors was 7.8 years with a 5-year overall survival of $55 \%$. While some patients with a benign histology did receive chemotherapy and radiation, these modalities were mostly reserved for patients with malignant SFT.

The literature on spinal SFT is limited to case reports and small case series. Therefore, we cannot comment on its incidence, although it does appear to be a very rare diagnosis. Likewise, since existing reports include very small numbers of patients, it is difficult to determine epidemiology, ideal treatment, follow-up, and overall prognosis. However, based on our review of 82 patients, we can attempt to draw some conclusions.

Not surprisingly, most of the tumors occur in the thoracic spine, as is seen with other spine neoplasms. The intradural extramedullary compartment of the spine is the most common space to find these tumors, although they are also frequently reported in the extradural space and intramedullary space with an exophytic component. Imaging characteristics, particularly for extradural and intradural extramedullary tumors, tend to mimic meningiomas and/or nerve sheath tumors. The tumors can arise from any tissue, and their origin is not always evident on imaging or during surgery.

With regard to management, the overwhelming majority of patients are treated with surgery. Gross-total resection should be the goal and can be curative. Subtotally resected tumors tend to recur, although the number of patients who underwent STR in our series is small. There
TABLE 2. Demographics and tumor characteristics

\begin{tabular}{lc}
\hline \multicolumn{1}{c}{ Variable } & Value \\
\hline Age (yrs) & \\
\hline Mean (SD) & $49.8(15.4)$ \\
\hline Median (range) & $51.5(10-83)$ \\
\hline Sex & $44(56.4 \%)$ \\
\hline Male & $34(43.6 \%)$ \\
\hline Female & \\
\hline Level & $2(2.6 \%)$ \\
\hline Occipitocervical & $21(27.6 \%)$ \\
\hline Cervical & $3(3.9 \%)$ \\
\hline Cervicothoracic & $32(42.1 \%)$ \\
\hline Thoracic & $1(1.3 \%)$ \\
\hline Thoracolumbar & $11(14.5 \%)$ \\
\hline Lumbar & $1(1.3 \%)$ \\
\hline Lumbosacral & $1(1.3 \%)$ \\
\hline Sacral & $4(5.3 \%)$ \\
\hline Multiple & \\
\hline Compartment & $18(26.5 \%)$ \\
\hline Extradural & $5(7.4 \%)$ \\
\hline Extradural \& intradural extramedullary & $24(35.3 \%)$ \\
\hline Intradural extramedullary & $11(16.2 \%)$ \\
\hline Intradural extramedullary \& intramedullary & $10(14.7 \%)$ \\
\hline Intramedullary &
\end{tabular}

does not appear to be a role for adjuvant therapy for the benign SFT patient who has had a successful GTR. Adjuvant therapy for residual benign SFT is probably also of little benefit given the overall good prognosis of these tumors and the literature on SFTs in other locations suggesting that adjuvant therapy is of little value for benign SFT. Malignant SFT, on the other hand, appears to have a very poor prognosis regardless of intervention. There were very few malignant SFT cases in our series, but the patients in these cases all fared poorly. In these patients, strong consideration should be given to resection followed by adjuvant therapy, although it is not clear how much impact this will have on life expectancy.

Despite its benign histology, SFT can recur. This has been seen with SFTs in other nonspinal locations. Most patients in our series had very short follow-ups. Interestingly, the tumors that did recur seemed to do so late (2 years, 8.5 years, 5 and 9 years, and 14.5 years), suggesting that there may be value in the long-term follow-up of patients. In addition, 3 of the patients with spinal SFT had prior intracranial lesions and presented with spinal lesions consistent with drop metastases. All of these lesions had benign histologies. The lag time between intracranial disease and the diagnosis of spinal disease was 10 years, ${ }^{68} 14$ years, ${ }^{56}$ and 15 years. ${ }^{47}$

Solitary fibrous tumors in any location are much more common in adults than in children. In the pleura, it seems that less than $2 \%$ of SFTs occur in children. In addition, children have a lower incidence of malignant pleural SFT than adults do. ${ }^{45}$ Solitary fibrous tumors have been report- 
ed in other locations in children including the mesentery, ${ }^{75}$ scalp, ${ }^{61}$ kidney, ${ }^{77}$ orbit, ${ }^{41}$ shoulder, ${ }^{60}$ and brain. ${ }^{63}$ Prior to this report, the youngest reported patient with spinal SFT was 17 years of age ${ }^{30}$ and only 2 other reported patients were younger than 21 years of age. ${ }^{11,71}$ Our patient is the first documented preadolescent child with spinal SFT.

The major limitation of this paper is its reliance on previously published case reports and case series. These previous reports have all been selected for publication based on unusual features of the cases. There is inevitably publication bias that introduces selection bias to this report. In addition, previous authors have been selective about the information they have included in their reports. Unfortunately, given the rarity of SFTs of the spine, larger studies are unlikely to be performed.

\section{Conclusions}

Solitary fibrous tumors of the spine are rare neoplasms. No large series exist. However, given this review of reported cases, it appears that the majority of these lesions are benign and should be treated with GTR alone. Even under these circumstances, however, the tumors can recur and thus long-term follow-up should be considered.

\section{References}

1. Aftab S, Casey A, Tirabosco R, Kabir SR, Saifuddin A: Fat-forming solitary fibrous tumour (lipomatous haemangiopericytoma) of the spine: case report and literature review. Skeletal Radiol 39:1039-1042, 2010

2. Alston SR, Francel PC, Jane JA Jr: Solitary fibrous tumor of the spinal cord. Am J Surg Pathol 21:477-483, 1997

3. Arantes M, Honavar M, Vaz AR, Resende M, Pereira JR: Solitary fibrous tumor of the thoracic spine. Neurochirurgie 55:573-575, 2009

4. Basaran R, Kaksi M, Onoz M, Balkuv E, Sav A: Intradural solitary fibrous tumor of the lumbar spine: a distinctive case report. Case Rep Neurol Med 2015:708472, 2015

5. Bisceglia M, Galliani C, Giannatempo G, Lauriola W, Bianco M, D'Angelo V, et al: Solitary fibrous tumor of the central nervous system: a 15-year literature survey of 220 cases (August 1996-July 2011). Adv Anat Pathol 18:356-392, 2011

6. Boddaert G, Guiraudet P, Grand B, Venissac N, Le PimpecBarthes F, Mouroux J, et al: Solitary fibrous tumors of the pleura: a poorly defined malignancy profile. Ann Thorac Surg 99:1025-1031, 2015

7. Bohinski RJ, Mendel E, Aldape KD, Rhines LD: Intramedullary and extramedullary solitary fibrous tumor of the cervical spine. Case report and review of the literature. J Neurosurg 100 (4 Suppl Spine):358-363, 2004

8. Bouyer B, Guedj N, Lonjon G, Guigui P: Recurrent solitary fibrous tumour of the thoracic spine. A case-report and literature review. Orthop Traumatol Surg Res 98:850-853, 2012

9. Brigui M, Aldea S, Bernier M, Bennis S, Mireau E, Gaillard $\mathrm{S}$ : Two patients with a solitary fibrous tumor of the thoracic spinal cord. J Clin Neurosci 20:317-319, 2013

10. Bruder M, Tews D, Mittelbronn M, Capper D, Seifert V, Marquardt G: Intramedullary solitary fibrous tumor-a benign form of hemangiopericytoma? Case report and review of the literature. World Neurosurg 84:189.e7-189.e12, 2015

11. Brunori A, Cerasoli S, Donati R, Giangaspero F, Chiappetta F: Solitary fibrous tumor of the meninges: two new cases and review of the literature. Surg Neurol 51:636-640, 1999

12. Carneiro SS, Scheithauer BW, Nascimento AG, Hirose T, Davis DH: Solitary fibrous tumor of the meninges: a lesion distinct from fibrous meningioma. A clinicopathologic and immunohistochemical study. Am J Clin Pathol 106:217224, 1996

13. Caroli E, Salvati M, Orlando ER, Lenzi J, Santoro A, Giangaspero F: Solitary fibrous tumors of the meninges: report of four cases and literature review. Neurosurg Rev 27:246-251, 2004

14. Chen H, Zeng XW, Wu JS, Dou YF, Wang Y, Zhong P, et al: Solitary fibrous tumor of the central nervous system: a clinicopathologic study of 24 cases. Acta Neurochir (Wien) 154:237-248, 2012

15. Chick JF, Chauhan NR, Madan R: Solitary fibrous tumors of the thorax: nomenclature, epidemiology, radiologic and pathologic findings, differential diagnoses, and management. AJR Am J Roentgenol 200:W238-W248, 2013

16. Ciappetta P, D’Urso PI, Cimmino A, Ingravallo G, Rossi R, Colamaria A, et al: Intramedullary solitary fibrous tumor of dorsal spinal cord. Neuropathology 30:273-278, 2010

17. Cincu R, Rodriguez R, Perez A, Blanco T, Arrotegui I, Barcia C: Solitary fibrous tumor of the thoracic spine. J Neurosci Rural Pract 1:118-119, 2010

18. Demicco EG, Park MS, Araujo DM, Fox PS, Bassett RL, Pollock RE, et al: Solitary fibrous tumor: a clinicopathological study of 110 cases and proposed risk assessment model. Mod Pathol 25:1298-1306, 2012

19. DeVito N, Henderson E, Han G, Reed D, Bui MM, Lavey $\mathrm{R}$, et al: Clinical characteristics and outcomes for solitary fibrous tumor (SFT): a single center experience. PLoS One 10:e0140362, 2015

20. Donnellan RB, Govender D, Chite SH, Landers AT: An unusual presentation of solitary fibrous tumor. Spine (Phila Pa 1976) 25:749-751, 2000

21. Endo K, Komagata M, Ikegami H, Nishiyama M, Tanaka S, Imakiire A, et al: Dumbbell-type solitary fibrous tumor in the cervical spine. J Orthop Sci 8:428-431, 2003

22. Fargen KM, Opalach KJ, Wakefield D, Jacob RP, Yachnis AT, Lister JR: The central nervous system solitary fibrous tumor: a review of clinical, imaging and pathologic findings among all reported cases from 1996 to 2010. Clin Neurol Neurosurg 113:703-710, 2011

23. Giannini C, Rushing E, Hainfellner J: Haemangiopericytoma, in Louis D, Ohgaki H, Wiestler O, et al (eds): WHO Classification of Tumours of the Central Nervous System. Lyon: IARC Press, 2007

24. Guo G, Zhang X, Zhou ZH: Clinical characteristics of malignant solitary fibrous tumors of the kidney with thoracic vertebral metastasis. Int J Urol 19:177-178, 2012

25. Hasegawa T, Matsuno Y, Shimoda T, Hasegawa F, Sano T, Hirohashi S: Extrathoracic solitary fibrous tumors: their histological variability and potentially aggressive behavior. Hum Pathol 30:1464-1473, 1999

26. Hashimoto K, Miyamoto K, Hosoe H, Kawai G, Kikuike K, Shimokawa K, et al: Solitary fibrous tumor in the cervical spine with destructive vertebral involvement: a case report and review of the literature. Arch Orthop Trauma Surg 128:1111-1116, 2008

27. Hirakawa A, Miyamoto K, Hosoe H, Nishimoto Y, Shimokawa K, Shimizu K: Solitary fibrous tumor in the occipitocervical region: a case report. Spine (Phila Pa 1976) 29:E547E550, 2004

28. Hwang US, Kim SB, Jo DJ, Kim SM: Intramedullary solitary fibrous tumor of cervicothoracic spinal cord. J Korean Neurosurg Soc 56:265-268, 2014

29. Ishii K, Nakamura M, Matsumoto M, Mukai M, Toyama Y, Chiba K: Intramedullary solitary fibrous tumor of the spinal cord. J Orthop Sci 14:450-454, 2009

30. Jallo GI, Roonprapunt C, Kothbauer K, Freed D, Allen J, Epstein F: Spinal solitary fibrous tumors: a series of four patients: case report. Neurosurgery 57:E195, 2005

31. Kakimaru H, Matsusaki M, Sanada H, Iwata A, Uchio Y: 
Dumbbell-type spinal solitary fibrous tumor with paraplegia. Orthopedics 32:213, 2009

32. Kanahara T, Hirokawa M, Shimizu M, Terayama K, Nakamura E, Hino Y, et al: Solitary fibrous tumor of the spinal cord. Report of a case with scrape cytology. Acta Cytol 43:425-428, 1999

33. Kataoka H, Akiyama Y, Kubo S, Itoh H, Hamasuna R, Tajima $\mathrm{N}$, et al: Solitary fibrous tumor of the spinal nerve rootlet: case report and literature survey. Pathol Int 49:826-830, 1999

34. Kawamura M, Izawa K, Hosono N, Hirano H: Solitary fibrous tumor of the spinal cord: case report and review of the literature. Neurosurgery 55:433, 2004

35. Kim KA, Gonzalez I, McComb JG, Giannotta SL: Unusual presentations of cerebral solitary fibrous tumors: report of four cases. Neurosurgery 54:1004-1009, 2004

36. Klemperer P, Rabin CB: Primary neoplasms of the pleura: report of five cases. Arch Pathol (Chic) 11:385-412, 1931

37. Kobayashi K, Imagama S, Ito Z, Ando K, Ukai J, Muramoto A, et al: Recurrence of solitary fibrous tumor of the cervical spinal cord. Nagoya J Med Sci 76:217-223, 2014

38. Kuchelmeister K, Scheuerle A, Bohle RM, Kretschmer T, Richter HP, Schachenmayr W: Solitary fibrous tumor-a new differential diagnosis in surgical neuropathology. Report of two cases. Clin Neuropathol 16:270, 1997

39. Kurtkaya O, Elmaci I, Sav A, Pamir MN: Spinal solitary fibrous tumor: seventh reported case and review of the literature. Spinal Cord 39:57-60, 2001

40. Lavrador JP, Oliveira E, Neto L, Pimentel J, Francisco AF, Livraghi S: Dumbbell-shaped spinal solitary fibrous tumor: Combined approach and a review of the literature. Neurochirurgie 61:287-291, 2015

41. Lucci LM, Anderson RL, Harrie RP, Mamalis N, Coffin C, Crandall DC: Solitary fibrous tumor of the orbit in a child. Ophthal Plast Reconstr Surg 17:369-373, 2001

42. Malek AM, Weller SJ, Price DL Jr, Madsen JR: Solitary fibrous tumor presenting as a symptomatic intraspinal mass: case report. Neurosurgery 40:844-847, 1997

43. Mariniello G, Napoli M, Russo C, Briganti F, Giamundo A, Maiuri F, et al: MRI features of spinal solitary fibrous tumors. A report of two cases and literature review. Neuroradiol J 25:610-616, 2012

44. Mena H, Ribas JL, Pezeshkpour GH, Cowan DN, Parisi JE: Hemangiopericytoma of the central nervous system: a review of 94 cases. Hum Pathol 22:84-91, 1991

45. Merli L, Crocoli A, Boldrini R, Inserra A: Solitary fibrous tumor of the pleura in a 6-year-old boy. J Pediatr Surg 47:435-437, 2012

46. Metellus P, Bouvier C, Guyotat J, Fuentes S, Jouvet A, Vasiljevic A, et al: Solitary fibrous tumors of the central nervous system: clinicopathological and therapeutic considerations of 18 cases. Neurosurgery 60:715-722, 2007

47. Miyashita K, Hayashi Y, Fujisawa H, Hasegawa M, Yamashita J: Recurrent intracranial solitary fibrous tumor with cerebrospinal fluid dissemination. Case report. J Neurosurg 101:1045-1048, 2004

48. Montano N, Rigante L, Papacci F, Novello M, Lauriola L, Meglio M: Intradural extramedullary lesion of the conus medullaris. Solitary fibrous tumor. J Clin Neurosci 20:715, 765,2013

49. Mordani JP, Haq IU, Singh J: Solitary fibrous tumour of the spinal cord. Neuroradiology 42:679-681, 2000

50. Muñoz E, Prat A, Adamo B, Peralta S, Ramón y Cajal S, Valverde C: A rare case of malignant solitary fibrous tumor of the spinal cord. Spine (Phila Pa 1976) 33:E397-E399, 2008

51. Nagano A, Ohno T, Nishimoto Y, Oshima K, Shimizu K: Malignant solitary fibrous tumor of the lumbar spinal root mimicking schwannoma: a case report. Spine J 14:e17-e20, 2014

52. Nakamura Y, Okajima K, Otsuka F, Ohara K: Solitary fibrous tumor attached to the cervical vertebra. Dermatol Surg 33:500-504, 2007

53. Obara Y, Matsumoto M, Chiba K, Yabe H, Toyama Y, Mukai M: Solitary cervical fibrous tumor. Case illustration. J Neurosurg 98 (1 Suppl):111, 2003

54. Ogawa T, Moriyama E, Beck H, Sonobe H: Solitary fibrous tumor of the thoracic spinal cord. Neurol Med Chir (Tokyo) 45:371-374, 2005

55. Ogungbo B, Prakash S, Kulkarni G, Bradey N, Marks SM, Scoones D: Cervical intra-/extramedullary solitary fibrous tumour. Br J Neurosurg 19:254-257, 2005

56. Pakasa NM, Pasquier B, Chambonnière ML, Morrison AL, Khaddage A, Perret AG, et al: Atypical presentations of solitary fibrous tumors of the central nervous system: an analysis of unusual clinicopathological and outcome patterns in three new cases with a review of the literature. Virchows Arch 447:81-86, 2005

57. Perry A, Scheithauer BW, Nascimento AG: The immunophenotypic spectrum of meningeal hemangiopericytoma: a comparison with fibrous meningioma and solitary fibrous tumor of meninges. Am J Surg Pathol 21:1354-1360, 1997

58. Piana S, Putrino I, Cavazza A, Nigrisoli E: Solitary fibrous tumor of the spinal nerve rootlet: report of a case mimicking schwannoma. Arch Pathol Lab Med 128:335-337, 2004

59. Pizzolitto S, Falconieri G, Demaglio G: Solitary fibrous tumor of the spinal cord: a clinicopathologic study of two cases. Ann Diagn Pathol 8:268-275, 2004

60. Rakheja D, Wilson KS, Meehan JJ, Schultz RA, Maale GE, Timmons CF: Extrapleural benign solitary fibrous tumor in the shoulder of a 9-year-old girl: case report and review of the literature. Pediatr Dev Pathol 7:653-660, 2004

61. Rizk T, Awada A, Sebaaly A, Hourani R: Solitary fibrous tumor of the scalp in a child. J Neurosurg Pediatr 11:79-81, 2013

62. Robert T, Duc C, San Millán Ruíz D, Morard M: Solitary fibrous tumour with intramedullary component: case report and review of the literature. Neurol Neurochir Pol 48:144149,2014

63. Rubacha M, Tubbs RS, Li R, Rocque B, Blount JP: Pediatric central nervous system solitary fibrous tumor: case report. Childs Nerv Syst 31:2379-2381, 2015

64. Sade R, Çakır M, Ogul H, Çalıkoğlu Ç, Kantarci M: Very rare reason of spinal cord compression: solitary fibrous tumor. Spine J 15:1158-1159, 2015

65. Sebaaly A, Raffoul L, Moussa R: Solitary fibrous tumor of the lumbar spine: the great mimicker-report of the fifth case. Case Rep Orthop 2014:852830, 2014

66. Shin DA, Kim SH, Yoon DH, Kim TS: A dumbbell-shaped solitary fibrous tumor of the cervical spinal cord. Yonsei Med J 49:167-170, 2008

67. Son S, Lee SG, Jeong DH, Yoo CJ: Malignant solitary fibrous tumor of tandem lesions in the skull and spine. J Korean Neurosurg Soc 54:246-249, 2013

68. Takenouchi T, Pannullo SC, Stieg PE, Lavi E: Solitary fibrous tumor with multiple intracranial and spinal lesions: case report. Neurosurgery 68:E1148-E1151, 2011

69. Tihan T, Viglione M, Rosenblum MK, Olivi A, Burger PC: Solitary fibrous tumors in the central nervous system. A clinicopathologic review of 18 cases and comparison to meningeal hemangiopericytomas. Arch Pathol Lab Med 127:432-439, 2003

70. Tomek M, Bravi I, Mendoza N, Alsafi A, Mehta A, Molinaro L, et al: Spinal extradural solitary fibrous tumor with retiform and papillary features. Ann Diagn Pathol 17:281-287, 2013

71. Tsutsumi N, Kojima Y, Nishida K, Maeno K, Kakutani K, Kawakami F, et al: Surgical treatment for recurrent solitary fibrous tumor invading atlas. Head Neck 36:E121-E124, 2014

72. Vassal F, Manet R, Forest F, Camdessanche JP, Péoc'h M, 
Nuti C: Solitary fibrous tumors of the central nervous system: report of five cases with unusual clinicopathological and outcome patterns. Acta Neurochir (Wien) 153:377-384, 2011

73. Vorster SJ, Prayson RA, Lee JH: Solitary fibrous tumor of the thoracic spine. Case report and review of the literature. $\mathbf{J}$ Neurosurg 92 (2 Suppl):217-220, 2000

74. Walker CT, Amene CS, Pannell JS, Santiago-Dieppa DR, Rennert RC, Hansen LA, et al: Hemorrhagic intramedullary solitary fibrous tumor of the conus medullaris: case report. $\mathbf{J}$ Neurosurg Spine 23:438-443, 2015

75. Wang H, Shen D, Hou Y: Malignant solitary tumor in a child: a case report and review of the literature. J Pediatr Surg 46:e5-e9, 2011

76. Wang XQ, Zhou Q, Li ST, Liao CL, Zhang H, Zhang BY: Solitary fibrous tumors of the central nervous system: clinical features and imaging findings in 22 patients. J Comput Assist Tomogr 37:658-665, 2013

77. Wu WW, Chu JT, Romansky SG, Shane L: Pediatric renal solitary fibrous tumor: report of a rare case and review of the literature. Int J Surg Pathol 23:34-47, 2015

78. Wu Y, Huang B, Liang C: Solitary fibrous tumor of filum terminale. Acta Radiol Short Rep 1:20, 2012

79. Wushou A, Jiang YZ, Liu YR, Shao ZM: The demographic features, clinicopathologic characteristics, treatment outcome and disease-specific prognostic factors of solitary fibrous tumor: a population-based analysis. Oncotarget 6:4187541883, 2015
80. Yuan L, Chen X, Tian H, Chen S: Dumbbell-shaped intraspinal solitary fibrous tumor extending into the thoracic cavity. Clin Neuropathol 33:91-93, 2014

81. Zaldivar-Jolissaint JF, Mascott C, Gugliotta M, Duff JM: Extensive bone remodeling from a solitary fibrous tumor of the cervical spine. Spine J 16:e699-e700, 2016

\section{Disclosures}

The authors report no conflict of interest concerning the materials or methods used in this study or the findings specified in this paper.

\section{Author Contributions}

Conception and design: Albert. Acquisition of data: both authors. Analysis and interpretation of data: Albert. Drafting the article: Albert. Critically revising the article: both authors. Reviewed submitted version of manuscript: Albert. Approved the final version of the manuscript on behalf of both authors: Albert. Statistical analysis: Albert. Study supervision: Albert.

\section{Correspondence}

Gregory W. Albert, Division of Neurosurgery, Department of Neurosurgery, Arkansas Children's Hospital, 1 Children's Way, Slot 838, Little Rock, AR 72202. email: galbert2@uams.edu. 